\title{
IDENTITIES WITH DERIVATIONS AND AUTOMORPHISMS ON SEMIPRIME RINGS
}

JOSO VUKMAN

Received 31 May 2004 and in revised form 17 January 2005

The purpose of this paper is to investigate identities with derivations and automorphisms on semiprime rings. A classical result of Posner states that the existence of a nonzero centralizing derivation on a prime ring forces the ring to be commutative. Mayne proved that in case there exists a nontrivial centralizing automorphism on a prime ring, then the ring is commutative. In this paper, some results related to Posner's theorem as well as to Mayne's theorem are proved.

We investigate identities with derivations and automorphisms on semiprime rings. We prove, for example, that in case there exist a derivation $D: R \rightarrow R$ and an automorphism $\alpha: R \rightarrow R$, where $R$ is a 2-torsion-free semiprime ring, such that $[D(x) x+x \alpha(x), x]=0$ holds for all $x \in R$, then $D$ and $\alpha-I$, where $I$ denotes the identity mapping, map $R$ into its center. Throughout this paper, $R$ will represent an associative ring with center $Z(R)$. A ring $R$ is 2-torsion-free in case $2 x=0$ implies that $x=0$ for any $x \in R$. As usual, we write $[x, y]$ for $x y-y x$ and make use of the commutator identities $[x y, z]=[x, z] y+$ $x[y, z],[x, y z]=[x, y] z+y[x, z], x, y, z \in R$. We denote by $I$ the identity mapping of a ring $R$. An additive mapping $D: R \rightarrow R$ is called a derivation if $D(x y)=D(x) y+x D(y)$ holds for all pairs $x, y \in R$. Let $\alpha$ be an automorphism of a ring $R$. An additive mapping $D: R \rightarrow R$ is called an $\alpha$-derivation if $D(x y)=D(x) \alpha(y)+x D(y)$ holds for all pairs $x, y \in R$. Note that the mapping $D=\alpha-I$ is an $\alpha$-derivation. Of course, the concept of $\alpha$-derivation generalizes the concept of derivation, since $I$-derivation is a derivation. We denote by $C$ the extended centroid of a semiprime ring $R$ and by $Q$ Martindale ring of quotients. For the explanation of the extended centroid of a semiprime ring $R$ and the Martindale ring of quotients, we refer the reader to [1]. A mapping $f$ of $R$ into itself is called centralizing on $R$ if $[f(x), x] \in Z(R)$ holds for all $x \in R$; in the special case when $[f(x), x]=0$ holds for all $x \in R$, the mapping $f$ is said to be commuting on $R$. The history of commuting and centralizing mappings goes back to 1955 when Divinsky [5] proved that a simple Artinian ring is commutative if it has a commuting nontrivial automorphism. Two years later, Posner [8] has proved that the existence of a nonzero centralizing derivation on a prime ring forces the ring to be commutative (Posner's second theorem). Luh [6] generalized the Divinsky result, we have just mentioned above, to arbitrary prime 


\section{Identities with derivations and automorphisms}

rings. Mayne [7] proved that in case there exists a nontrivial centralizing automorphism on a prime ring, then the ring is commutative (Mayne's theorem). A result of Brešar [2], which states that every additive commuting mapping of a prime ring $R$ is of the form $x \mapsto \lambda x+\zeta(x)$ where $\lambda$ is an element of $C$ and $\zeta: R \rightarrow C$ is an additive mapping, should be mentioned. A mapping $f: R \rightarrow R$ is called skew-centralizing on $R$ if $f(x) x+x f(x) \in Z(R)$ holds for all $x \in R$; in particular, if $f(x) x+x f(x)=0$ holds for all $x \in R$, then it is called skew-commuting on $R$. Brešar [3] has proved that if $R$ is a 2-torsion-free semiprime ring and $f: R \rightarrow R$ is an additive skew-commuting mapping on $R$, then $f=0$.

First, we list three lemmas which will be needed in the sequel.

Lemma 1 [11, Lemma 1]. Let $R$ be a semiprime ring. Suppose that the relation $a x b+b x c=0$ holds for all $x \in R$ and some $a, b, c \in R$. In this case, $(a+c) x b=0$ is satisfied for all $x \in R$.

Lemma 2 [12, Lemma 1.3]. Let $R$ be a semiprime ring. Suppose that there exists $a \in R$ such that $a[x, y]=0$ holds for all pairs $x, y \in R$. In this case, $a \in Z(R)$.

Lemмa 3. Let $R$ be a semiprime ring and let $f: R \rightarrow R$ be an additive mapping. If either $f(x) x=0$ or $x f(x)=0$ holds for all $x \in R$, then $f=0$.

Proof. We can restrict our attention to the case

$$
f(x) x=0, \quad x \in R
$$

because of left-right symmetry. The linearization of the above relation gives

$$
f(x) y+f(y) x=0, \quad x, y \in R
$$

The substitution of $y^{2}$ for $y$ in the above relation gives

$$
f(x) y^{2}+f\left(y^{2}\right) x=0, \quad x, y \in R .
$$

Right multiplication of (2) by $y$ gives

$$
f(x) y^{2}+f(y) x y=0, \quad x, y \in R
$$

Subtracting (4) from (3), we obtain

$$
f\left(y^{2}\right) x-f(y) x y=0, \quad x, y \in R
$$

Putting in the above relation $x f(y)$ for $x$, we obtain, because of (1),

$$
f\left(y^{2}\right) x f(y)=0, \quad x, y \in R
$$

Right multiplication of (5) by $f(y)$ gives, because of the above relation, $f(y) x y f(y)=$ $0, x, y \in R$, which leads to $y f(y) x y f(y)=0, x, y \in R$, whence it follows that

$$
x f(x)=0, \quad x \in R .
$$


Right multiplication of the relation (2) by $f(x)$ gives, because of the above relation, $f(x) y f(x)=0, x, y \in R$, whence it follows that $f=0$, which completes the proof.

We are ready for our first result.

Theorem 4. Let $R$ be a semiprime ring. Suppose that there exist a derivation $D: R \rightarrow R$ and an automorphism $\alpha: R \rightarrow R$, such that the mapping $x \rightarrow D(x)+\alpha(x)$ is commuting on $R$. In this case, $D$ and $\alpha-I$ map $R$ into $Z(R)$.

At the end of the proof of the result above, we will need the result below.

Theorem 5 [9, Proposition 2.3]. Let $R$ be a semiprime ring and let $D: R \rightarrow R$ be a commuting $\alpha$-derivation on $R$. In this case, $D$ maps $R$ into $Z(R)$.

We will need also the result below which is a special case of [2, Proposition 3.1].

Theorem 6. Let $R$ be a 2-torsion-free semiprime ring and let $f: R \rightarrow R$ be an additive centralizing mapping on $R$. In this case, $f$ is commuting on $R$.

Proof of Theorem 4. The linearization of the relation

$$
[D(x)+\alpha(x), x]=0, \quad x \in R
$$

gives

$$
[D(x)+\alpha(x), y]+[D(y)+\alpha(y), x]=0, \quad x, y \in R \text {. }
$$

Putting in the above relation $y x$ for $y$ and applying the relation (8), we obtain

$$
\begin{aligned}
0= & {[D(x)+\alpha(x), y] x+[D(y) x+y D(x)+\alpha(y) \alpha(x), x] } \\
= & {[D(x)+\alpha(x), y] x+[D(y), x] x+[y, x] D(x)+y[D(x), x] } \\
& +[\alpha(y), x] \alpha(x)+\alpha(y)[\alpha(x), x], \quad x, y \in R .
\end{aligned}
$$

We therefore have

$$
\begin{aligned}
{[D(x)+} & \alpha(x), y] x+[D(y), x] x \\
& +[y, x] D(x)+y[D(x), x]+[\alpha(y), x] \alpha(x)+\alpha(y)[\alpha(x), x]=0, \quad x, y \in R .
\end{aligned}
$$

According to relations (8) and (9), one can replace in the above relation $y[D(x), x]$ by $-y[\alpha(x), x]$ and $[D(x)+\alpha(x), y] x+[D(y), x] x$ by $-[\alpha(y), x] x$ which gives

$$
[\alpha(y), x] G(x)+G(y)[\alpha(x), x]+[y, x] D(x)=0, \quad x, y \in R,
$$

where $G(x)$ denotes $\alpha(x)-x$. Putting in the above relation $x y$ for $y$, we obtain, after some calculation,

$$
\begin{gathered}
{[\alpha(x), x] \alpha(y) G(x)+\alpha(x)[\alpha(y), x] G(x)+G(x) \alpha(y)[\alpha(x), x]} \\
+x G(y)[\alpha(x), x]+x[y, x] D(x)=0, \quad x, y \in R .
\end{gathered}
$$




\section{Identities with derivations and automorphisms}

Multiplying the relation (12) from the left side by $x$, subtracting the relation so obtained from the above relation and replacing $\alpha(y)$ by $y$, we obtain (note that $[\alpha(x), x]=$ $[G(x), x], x \in R)$

$$
[G(x), x] y G(x)+G(x)[y, x] G(x)+G(x) y[G(x), x]=0, \quad x, y \in R,
$$

which reduces to

$$
x G(x) y G(x)+G(x) y(-G(x) x)=0, \quad x, y \in R .
$$

Applying Lemma 1, the above relation gives

$$
[G(x), x] y G(x)=0, \quad x, y \in R .
$$

Putting in the above relation $y x$ for $y$, then multiplying the relation (16) from the right side by $x$, then subtracting the relations so obtained one from another, we obtain $[G(x)$, $x] y[G(x), x]=0, x, y \in R$, which gives

$$
[G(x), x]=0, \quad x \in R
$$

We therefore have $[\alpha(x), x]=0, x \in R$, which gives, together with the relation (8),

$$
[D(x), x]=0, \quad x \in R
$$

We have therefore proved that $G$ and $D$ are both commuting on $R$. Now Theorem 5 completes the proof of the theorem.

Corollaries 7 and 8 are related to Posner's second theorem as well as to Mayne's theorem.

Corollary 7. Let $R$ be a 2-torsion-free semiprime ring. Suppose that there exist a derivation $D: R \rightarrow R$ and an automorphism $\alpha: R \rightarrow R$, such that the mapping $x \rightarrow D(x)+\alpha(x)$ is centralizing on $R$. In this case, $D$ and $\alpha-I$ map $R$ into $Z(R)$.

Proof. The proof is an immediate consequence of Theorems 6 and 4 .

COROLlaRY 8. Let $R$ be a noncommutative prime ring of characteristic different from two. Suppose that there exist a derivation $D: R \rightarrow R$ and an automorphism $\alpha: R \rightarrow R$, such that the mapping $x \rightarrow D(x)+\alpha(x)$ is centralizing on $R$. In this case, $D=0$ and $\alpha=I$.

Thaheem [9] has proved that in case we have derivations $D, G: R \rightarrow R$, where $R$ is a semiprime ring, satisfying the relation $D(x) x+x G(x)=0$, for all $x \in R$, then both derivations map $R$ into its center and $D=-G$ (see also [10]).

In the same paper, Thaheem raised the question about a solution of the equation

$$
f(x) x+x g(x)=0, \quad x \in R
$$

where $f$ and $g$ are additive mappings of a semiprime ring $R$ into itself.

The result below proved by Brešar [4] gives the answer to Thaheem's question in case $R$ is a prime ring. 
Theorem 9 [4, Corollary 4.9]. Let $R$ be a prime ring and let $f, g: R \rightarrow R$ be additive mappings satisfying the relation (19) for all $x \in R$. In this case, there exist $a \in Q$ and an additive mapping $\varsigma: R \rightarrow C$ such that

$$
f(x)=x a+\varsigma(x), \quad g(x)=-a x-\varsigma(x)
$$

for all $x \in R$.

We point out that the identity (19) generalizes both concepts, the concept of commuting and the concept of skew-commuting mappings.

Our next result is related to Thaheem's question mentioned above.

Theorem 10. Let $R$ be a semiprime ring. Suppose that there exist a derivation $D: R \rightarrow R$ and an automorphism $\alpha: R \rightarrow R$, such that $D(x) x+x(\alpha(x)-x)=0$ holds for all $x \in R$. In this case, $D=0$ and $\alpha=I$.

Proof. We have the relation

$$
D(x) x+x G(x)=0, \quad x \in R,
$$

where $G(x)$ stands for $\alpha(x)-x$. The linearization of the above relation gives

$$
D(x) y+D(y) x+x G(y)+y G(x)=0, \quad x, y \in R .
$$

Putting in the above relation $y x$ for $y$ and applying (21), we obtain

$$
D(x) y x+D(y) x^{2}+x G(y) \alpha(x)+x y G(x)=0, \quad x, y \in R
$$

Right multiplication of the relation (22) by $x$ gives

$$
D(x) y x+D(y) x^{2}+x G(y) x+y G(x) x=0, \quad x, y \in R .
$$

Subtracting the above relation from the relation (23), we obtain

$$
x G(y) G(x)+x y G(x)-y G(x) x=0, \quad x, y \in R .
$$

Putting in the above relation $x y$ for $y$ and applying (25), we obtain

$$
\begin{aligned}
0 & =x G(x) \alpha(y) G(x)+x^{2} G(y) G(x)+x^{2} y G(x)-x y G(x) x \\
& =x G(x) \alpha(y) G(x), \quad x, y \in R .
\end{aligned}
$$

We therefore have $x G(x) y G(x)=0, x, y \in R$, which gives $x G(x) y x G(x)=0, x, y \in R$, whence it follows that

$$
x G(x)=0, \quad x \in R
$$


From the above relation, one obtains according to Lemma 3 that $G(x)=0, x \in R$. In other words $\alpha=I$, which proves a part of the theorem. Now the relation (21) reduces to $D(x) x=0$, whence it follows, applying again Lemma 3, that $D=0$, which completes the proof of the theorem.

We are ready for our last result.

THeOREM 11. Let $R$ be a 2-torsion-free semiprime ring. Suppose that there exist a derivation $D: R \rightarrow R$ and an automorphism $\alpha: R \rightarrow R$, such that the mapping $x \rightarrow D(x) x+x \alpha(x)$ is commuting on $R$. In this case, $D$ and $\alpha-I$ map $R$ into $Z(R)$.

Proof. We have the relation

$$
[D(x) x+x \alpha(x), x]=0, \quad x \in R
$$

From the above relation, one easily obtains

$$
[A(x), y]+[D(x) y+D(y) x+x \alpha(y)+y \alpha(x), x]=0, \quad x, y \in R,
$$

where $A(x)$ stands for $D(x) x+x \alpha(x)$. Let in the relation above $y$ be $y x$. Then we have

$$
\begin{aligned}
0 & =[A(x), y x]+\left[D(x) y x+D(y) x^{2}+y D(x) x+x \alpha(y) \alpha(x)+y x \alpha(x), x\right] \\
& =[A(x), y x]+[(D(x) y+D(y) x) x, x]+[y A(x), x]+[x \alpha(y) \alpha(x), x] \\
& =[A(x), y] x+[D(x) y+D(y) x, x] x+[y, x] A(x)+[\alpha(y) \alpha(x), x], \quad x, y \in R .
\end{aligned}
$$

We therefore have

$$
[A(x), y] x+[D(x) y+D(y) x, x] x+[y, x] A(x)+x[\alpha(y) \alpha(x), x]=0, \quad x, y \in R .
$$

According to (29), one can replace in the above relation $[A(x), y] x+[D(x) y+D(y) x$, $x] x$ by $-[x \alpha(y)+y \alpha(x), x] x$. Thus we have

$$
\begin{aligned}
0= & -[x \alpha(y)+y \alpha(x), x] x+[y, x] A(x)+x[\alpha(y) \alpha(x), x] \\
= & x[\alpha(y), x] x-[y, x] \alpha(x) x-y[\alpha(x), x] x+[y, x] A(x) \\
& +x[\alpha(y), x] \alpha(x)+x \alpha(y)[\alpha(x), x], \quad x, y \in R .
\end{aligned}
$$

We therefore have

$$
x[\alpha(y), x] G(x)+[y, x] B(x)-y[\alpha(x), x] x+x \alpha(y)[\alpha(x), x]=0, \quad x, y \in R,
$$

where $G(x)$ and $B(x)$ denote $\alpha(x)-x$ and $D(x) x+[x, \alpha(x)]$, respectively. The substitution $x y$ for $y$ in the above relation gives

$$
\begin{aligned}
& x[\alpha(x), x] \alpha(y) G(x)+x \alpha(x)[\alpha(y), x] G(x)+x[y, x] B(x)-x y[\alpha(x), x] x \\
& \quad+x \alpha(x) \alpha(y)[\alpha(x), x]=0, \quad x, y \in R .
\end{aligned}
$$

Left multiplication of the relation (33) by $x$ gives

$$
x^{2}[\alpha(y), x] G(x)+x[y, x] B(x)-x y[\alpha(x), x] x+x^{2} \alpha(y)[\alpha(x), x]=0, \quad x, y \in R .
$$


Subtracting the above relation from the relation (34) and replacing $\alpha(y)$ by $y$, one obtains (note that $[\alpha(x), x]=[G(x), x], x \in R$ )

$$
x[G(x), x] y G(x)+x G(x)[y, x] G(x)+x G(x) y[G(x), x]=0, \quad x, y \in R .
$$

Collecting terms, the above relation we can write as

$$
-x^{2} G(x) y G(x)+x G(x) y G(x) x=0, \quad x, y \in R .
$$

The substitution of $y x$ for $y$ in the above relation gives

$$
-x^{2} G(x) y x G(x)+x G(x) y x G(x) x=0, \quad x, y \in R .
$$

Applying Lemma 1, one obtains, from the above relation,

$$
x[G(x), x] y x G(x)=0, \quad x, y \in R .
$$

Putting first in the above relation $y x$ for $y$, then multiplying the relation (39) from the right side by $x$, and then subtracting the relations so obtained one from another, we arrive at $x[G(x), x] y x[G(x), x]=0, x, y \in R$, whence it follows that

$$
x[\alpha(x), x]=0, \quad x \in R .
$$

Combining the above relation with the relation (28), one obtains

$$
[D(x), x] x=0, \quad x \in R .
$$

From the relation (40), one easily obtains

$$
x[\alpha(x), y]+x[\alpha(y), x]+y[\alpha(x), x]=0, \quad x, y \in R .
$$

The substitution $x y$ for $y$ in the above relation gives

$$
\begin{aligned}
0 & =x[\alpha(x), x y]+x[\alpha(x) \alpha(y), x]+x y[\alpha(x), x] \\
& =x^{2}[\alpha(x), y]+x \alpha(x)[\alpha(y), x]+x y[\alpha(x), x], \quad x, y \in R .
\end{aligned}
$$

We therefore have

$$
x^{2}[\alpha(x), y]+x \alpha(x)[\alpha(y), x]+x y[\alpha(x), x]=0, \quad x, y \in R .
$$

Multiplying the relation (42) from the left side by $x$ and subtracting the relation so obtained from the above relation, we obtain $x G(x)[\alpha(y), x]=0, x, y \in R$, which means that we have

$$
x G(x)[y, x]=0, \quad x, y \in R .
$$

Putting in the above relation $y z$ for $y$, we arrive at

$$
x G(x) y[z, x]=0, \quad x, y, z \in R .
$$




\section{Identities with derivations and automorphisms}

From the above relation, one obtains

$$
x G(x) y[z, w]+x G(w) y[z, x]+w G(x) y[z, x]=0, \quad x, y, z \in R .
$$

Putting in the above relation $[z, w] y x G(x)$ for $y$ and applying the relation (45), we obtain $(x G(x)[z, w]) y(x G(x)[z, w])=0, x, y, z, w \in R$, whence it follows that

$$
x G(x)[z, w]=0, \quad x, z, w \in R .
$$

From the above relation, it follows, according to Lemma 3, that

$$
G(x)[z, w]=0, \quad x, z, w \in R .
$$

From the above relation and Lemma 2, one can conclude that $G(x) \in Z(R)$ for any $x \in R$. In other words, $\alpha-I$ maps $R$ into $Z(R)$, which completes part of the proof. Using a similar approach, one can prove that $D$ maps $R$ into $Z(R)$ starting from the relation (41). The proof of the theorem is complete.

\section{Acknowledgments}

The author thanks the referees for helpful comments and suggestions. This research has been supported by the Research Council of Slovenia.

\section{References}

[1] K. I. Beidar, W. S. Martindale III, and A. V. Mikhalev, Rings with Generalized Identities, Monographs and Textbooks in Pure and Applied Mathematics, vol. 196, Marcel Dekker, New York, 1996.

[2] M. Brešar, Centralizing mappings and derivations in prime rings, J. Algebra 156 (1993), no. 2, 385-394.

[3] _ On skew-commuting mappings of rings, Bull. Austral. Math. Soc. 47 (1993), no. 2, 291296.

[4] - On generalized biderivations and related maps, J. Algebra 172 (1995), no. 3, 764-786.

[5] N. Divinsky, On commuting automorphisms of rings, Trans. Roy. Soc. Canada. Sect. III. (3) 49 (1955), 19-22.

[6] J. Luh, A note on commuting automorphisms of rings, Amer. Math. Monthly 77 (1970), 61-62.

[7] J. H. Mayne, Centralizing automorphisms of prime rings, Canad. Math. Bull. 19 (1976), no. 1, 113-115.

[8] E. C. Posner, Derivations in prime rings, Proc. Amer. Math. Soc. 8 (1957), 1093-1100.

[9] A. B. Thaheem and M. S. Samman, A note on $\alpha$-derivations on semiprime rings, Demonstratio Math. 34 (2001), no. 4, 783-788.

[10] J. Vukman, Identities with derivations on rings and Banach algebras, to appear in Glasnik Matematicki.

[11] Centralizers on semiprime rings, Comment. Math. Univ. Carolin. 42 (2001), no. 2, 237-245.

[12] B. Zalar, On centralizers of semiprime rings, Comment. Math. Univ. Carolin. 32 (1991), no. 4, 609-614.

Joso Vukman: Department of Mathematics, Faculty of Education, University of Maribor, Koroška cesta 160, 2000 Maribor, Slovenia

E-mail address: joso.vukman@uni-mb.si 


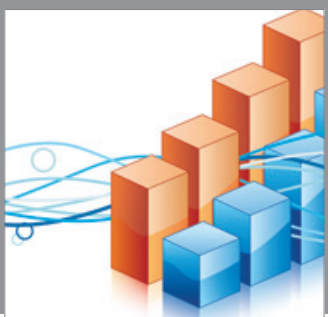

Advances in

Operations Research

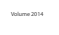

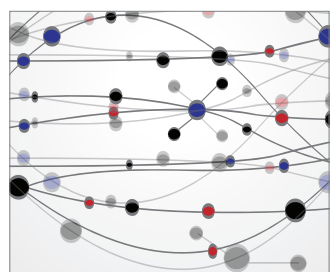

\section{The Scientific} World Journal
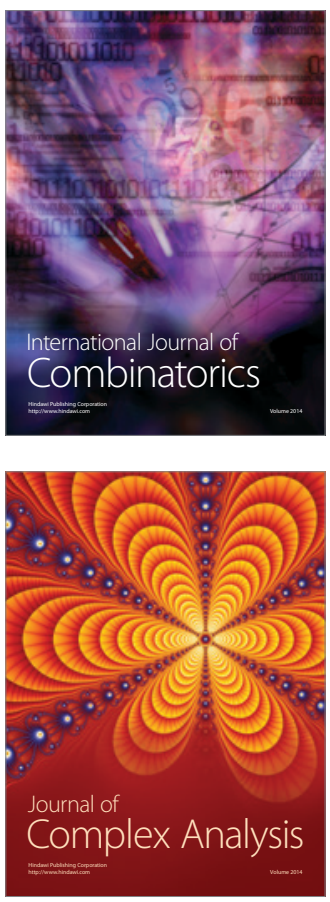

International Journal of

Mathematics and

Mathematical

Sciences
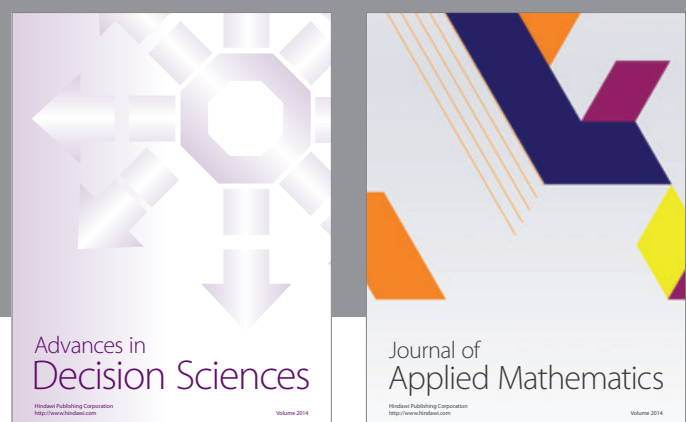

Journal of

Applied Mathematics
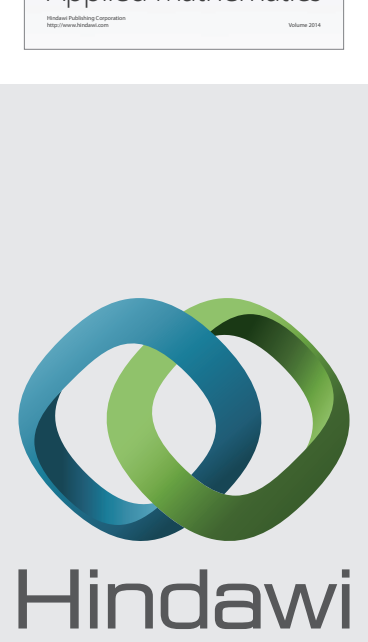

Submit your manuscripts at http://www.hindawi.com
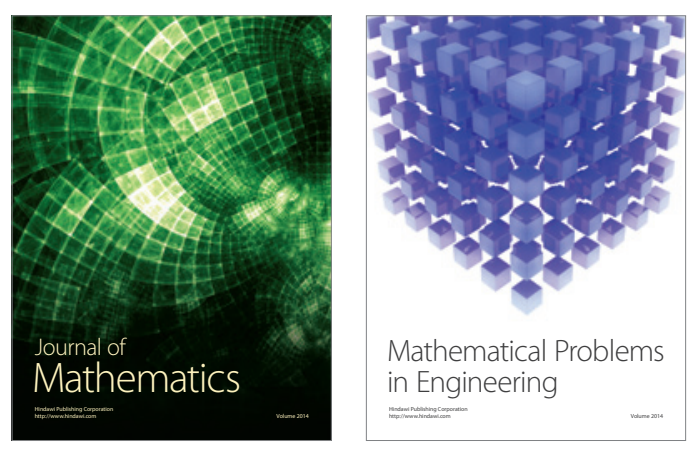

Mathematical Problems in Engineering
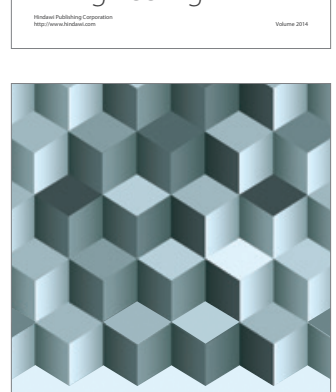

Journal of

Function Spaces
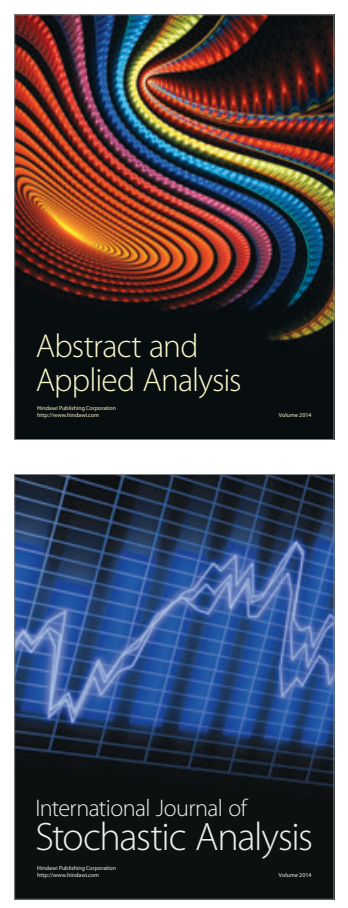

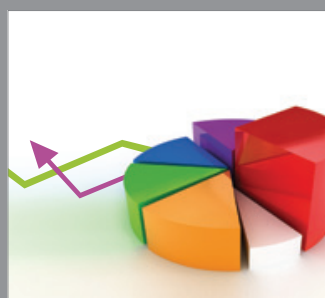

ournal of

Probability and Statistics

Promensencen
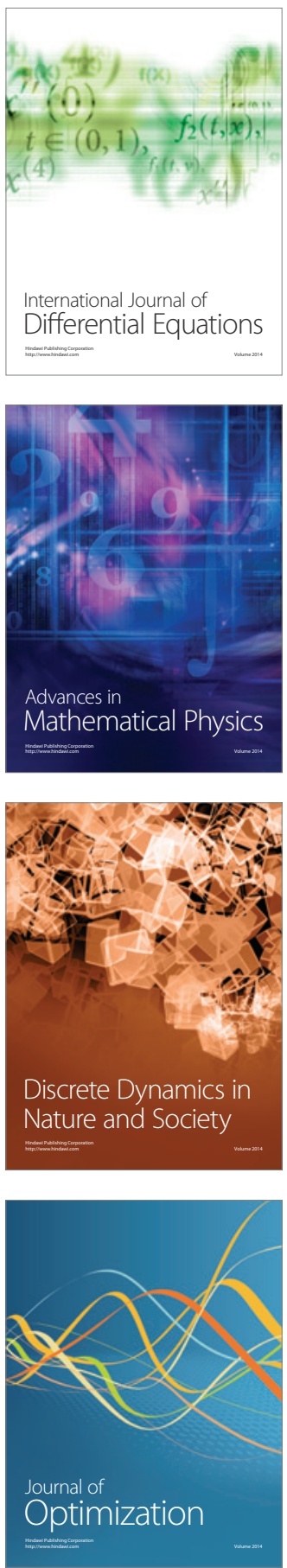